\title{
Malformación genital en pacientes con hiperplasia suprarrenal virilizante congénita. Corrección quirúrgica
}

\author{
Francisco Ossandón $\mathrm{C.}^{1}$; Ximena Vivanco W..$^{1}$; Ximena Cuello A. ${ }^{1}$; \\ Lydia Tellerías $\mathrm{C}^{+}$; Ingrid Bravo $\mathbf{A}^{2}$
}

\section{Genital reconstruction in congenital adrenal hyperplasia}

\begin{abstract}
Surgical results of genital reconstruction in 15 patients with congenital adrenal hyperplasia which were seen in a metropolitan general hospital at Santiago, Chile, along the last 20 vears are described. Blood 17 hydroxiprogesterone and urinary pregnanetriol together with sexual positive cromatin were the most useful tests to confirm the clinical diagnosis. Urinary 17 Ketosteroids were not diagnostic in two cases. Preoperative evaluation to define anatomy was done with pelvic ultrasound, genitography and cystoscopv. They were all useful, speciallv cystoscopy if it was done bv experienced persons. Surgical reconstruction of female external genitals was done in 14 girls at childhood with good aesthetic and functional results on the late controls. There was no introital estenosis at followup of cases that reached aduithood, except for one case of small bowel vaginoplasty, which will require further surgery. Male genitalia were asigned to one patient, because his problem was diagnosed late, at age five years, and fernale sex organs were then extirpated. The patient has good social sex identity but retains many feminine phenothypical elements.
\end{abstract}

(Key words: Congenital adrenal hyperplasia, genitoplasty.)

En la mujer, la causa más frecuente de genitales ambiguos es la hiperplasia suprarrenal virilizante congénita (HSVC). El tratamiento de estas pacientes presenta dos grandes desafíos: 1a insuficiencia suprarrenal con pérdida de sodio, que puede poner en peligro la vida y requiere un aporte adecuado $y$ permanente de glucocorticoides y mineralocorticoides, y, por otra parte, la reparación quinúrgica de las malformaciones genitales. En las pacientes con HSVC la alteración genital depende del grado de virilización producido en el período fetal. Este va desde una simple hipertrofia del clítoris hasta genitales totalmente virilizados, con un pene de aspecto nomal, ausencia de tercio inferior de vagina $y$ un escroto plano, en el que llama la atención

1. Universidad de Chile, División Ciencias Médicas Occidente, Departamento de Pediatria y Cirugía Infantil, Servicio de Cirugia Infantil y Pediatría, Unidad de Endocrinologia, Hospital San Juan de Dios.

2. Interna de Medicina, Ayudante-alumno. Unidad de Cirugía Infantil, División Ciencias Médicas Occidente, Universidad de Chile. la ausencia de gónadas. Sin embargo, la forma más común es la virilización parcial, en la que persiste el seno urogenital, al cual desemboca la vagina cerca de su extremo distal o bien en posición muy alta, por encima del esfinter externo de la uretra, último caso en que se requiere reqlizar procedimientos quirúrgicos más complejos para evitar daños a la continencia vesical (figura 1).

La corrección quirúrgica oportuna y adecuada de los genitales es indispensable para que el sexo de crianza del paciente se desenvuelva sin dudas ni ambigüedades por parte de los padres, de modo que la niffa injeie, desde que tiene noción de sus genitales, una identificación anatómica y psicológica con el rol sexual femenino.

A lo largo de los últimos 20 afios se ha producido un cambio respecto a la forma y oportunidad de abordar la corrección quirúrgica de los genitales. Se ha abandonado la clitoridectomía reemplazándola por plastía reductora del clitoris, intentando preservar la importante función sexual que tiene este órgano. La plastía de la vulva se hace más precozmente y el tiempo de 


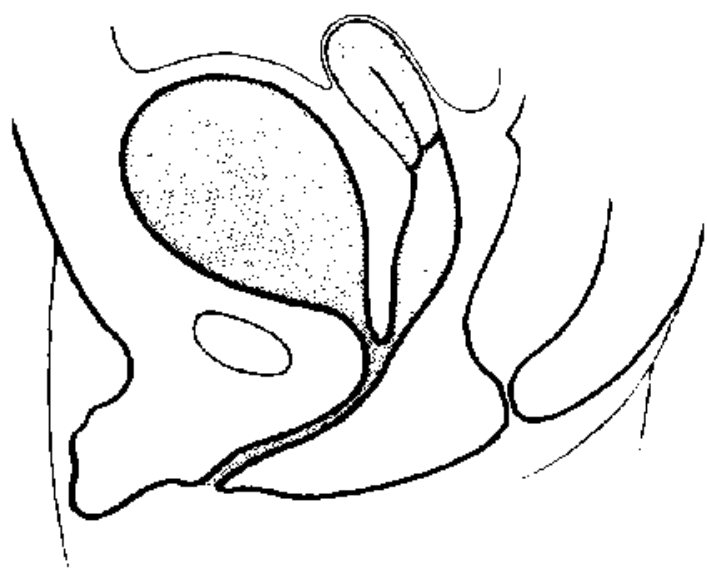

Figura 1: Vagina de desembogadura ala en el seno urogenita! por encima del esfínter estriado de la uretra.

la reparación vaginal varía según la anatomía de la malformación $y$ las diferentes conductas quirúrgicas. En todo caso el objetivo es asegurar a la niña menstruaciones nomales por un orificio vaginal y no por un seno urogenital, común con la vía urinaria.

En esta revisión se describe la casuística del Servicio de Cirugía Pediátrica y la Unidad de Endocrinologia del Hospital San Juan de Dios en los últimos 20 años, los resultados estéticos, funcionales y psicológicos en los padres y pacientes operados.

\section{Material y Métodos}

Se analizason retrospectivamente 15 casos de HSVC, controlados en la unidad de endocrinología infantil del Hospital San Juan de Dios entre los años 1969 y 1991 y se les realizó un seguimiento prospectivo, postoperaturio tardio, mediante una entrevista y examen clínico. Todas las pacientes se evaluaron con anamnesis, exarnen físico, examen genital externo y tacto rectal.

Los exámenes de laboratorio consistieron en determinación de la cromatina nuclear en los quince casos, 17 ketoesteroides urinatios (17 KTS) en once niñas y pregnantriol (Pngt) en orina de 24 horas en cinco; 17 hidroxiprogesterona (17 OHP) en diez pacientes y cortisol sanguíneo en seis; dehidroepiandrosterona sulfato plasmática (DHEAS) en 7 casos. Todas las determinaciones plasmáticas fueron realizadas por radioinmunoensayo. En dos pacientes se realizó examen del cariotipo. Para precisar la anatomía se realizó cistoscopja, genitograma y ecotomografía pelviana en la mayoría de los casos. Se buscó malformaciones renales asociadas con ecotomografía renal y pielografía de eliminación.
Las pacientes fueron preparadas para las operaciones con corticoides intramusculares y endovenosos, manteniendo dosis altas de ellos por al menos 24 horas de postoperatorio. Este tratamiento se prolongó cuando se estimó que había aún factores de estrés derivados de la cirugia.

Las técnicas quirúrgicas en la vulva vartaron de acuerdo a la época en que se réalizó el diagnóstico de las enfermas. Antes de 1986 sc realizó clitoridectom ía inicjal exclusiva. Posteriormente se realizó genitoplastía con plastía clitoridiana, que consistió en reducir el tamaño del clítoris seccionando los cuerpos cavernosos sin extirpar el glande, al que se le preservó la inervación e irrigación. El tamaño del glande fue reducido recortándolo y luego se suturó sobre el muñón residual de los cuerpos cavernosos. La vulva se corrigió abriendo el seno urogenital y tallando dos colgajos con el prepucio del clítoris, los que se descendieron a ambos lados del seno urogenital abierto, para constituir tos labios menores (figura 2).

Se realizaron 3 tipos de correcciones vaginales. La más simple y frecuente consistió en tallar un colgajo de piel, de base posterior a la horquilla vulvar, el que se avanzó abriendo el introito estenótico de la vagina por una incisión longitudinal en la pared posterior vaginal. Al suturar el colgajo sobre el defecto así creado se amplió el introito y la parte estrecha de la vagina. En estos casos la uretra quedó algo hipospádica. En las pacientes en que ta desembocadura de la vagina era alta en el seno urogenital, se realizó un descenso vaginal por vía abdominal, disecando la vagina e interrumpiendo su comunicación con el seno urogenital. Como la vagina que así se obtuvo era muy corta, se interpuso un trozo de intestino para elongarla y comunicarla al neointroito vaginal (figura 3 ).

\section{Resultados}

Nueve pacientes correspondian a la forma perdedora de sal de hiperplasia suprarrenal virilizante. En las otras seis no se encontró eviden. cia de pérđida salina.

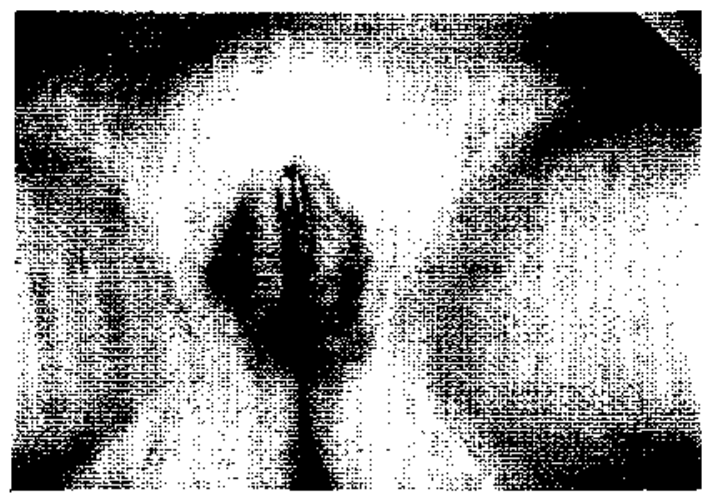

Figura 2: Aspecto pre y postoperatorio de genitoplastia precoz. 


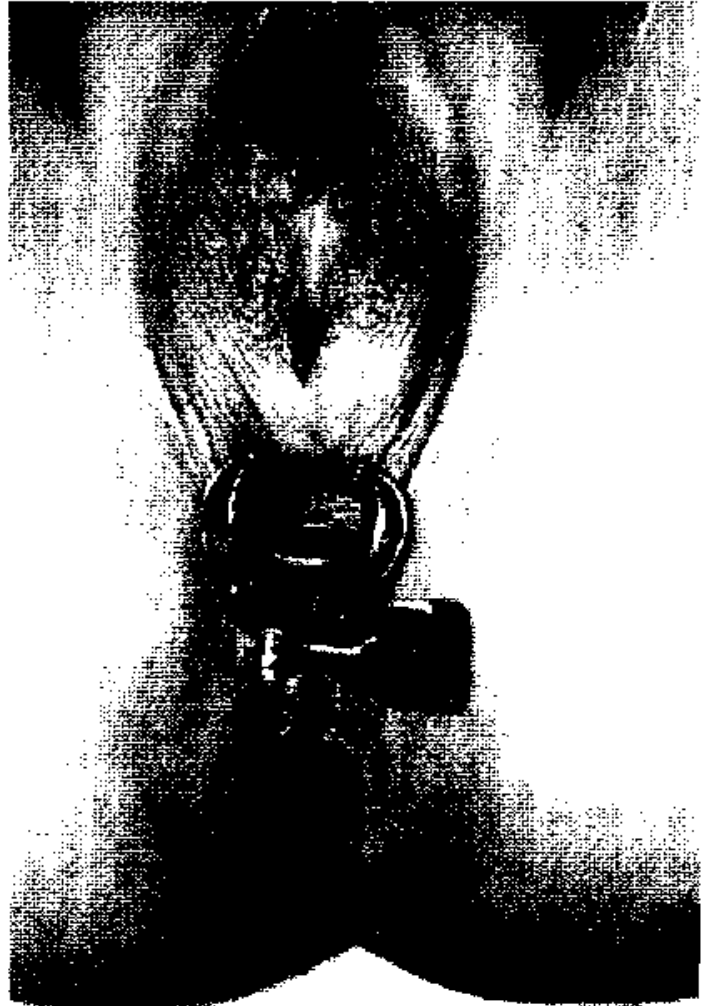

Figura 3: Neovagina con intestino. Aspecto externo con espéculo colocado en neovagina.

En catorce de las niñas el diagnóstico se hizo entre el período de recién nacido y los dos meses de edad y en una a los cinco años por pubertad precoz acompañada de supuesta criptorquidia bilateral, sin gónadas palpables (figura 4).

La cromatina nuclear fue positiva en todos los casos y el cariotipo fue $46 \mathrm{XX}$ en las dos pacientes en que se hizo. Los $17 \mathrm{KS}$ urinarios estaban aumentados en 9 casos y normales en 2 . En estos últimos dos pacientes el diagnóstico se confirmó por aumento de la $17 \mathrm{OHP}$. El pregnantriol uninario estaba elevado en tasas que confirmaron el diagnóstico en 4 casos. En una paciente en que no se detectó Pgnt, estaba aumentada la 17 OHP. Asimismo, en los diez casos en que se midieron concentraciones plasmáticas de $17 \mathrm{OHP}$, éstas resultaron elevadas, como también ocurrió con la DHEAS en cinco niñas en que se obtuvo el examen.

La ecotomografía pelviana demostró presencia de útero y ovarios en todos los casos. La en-
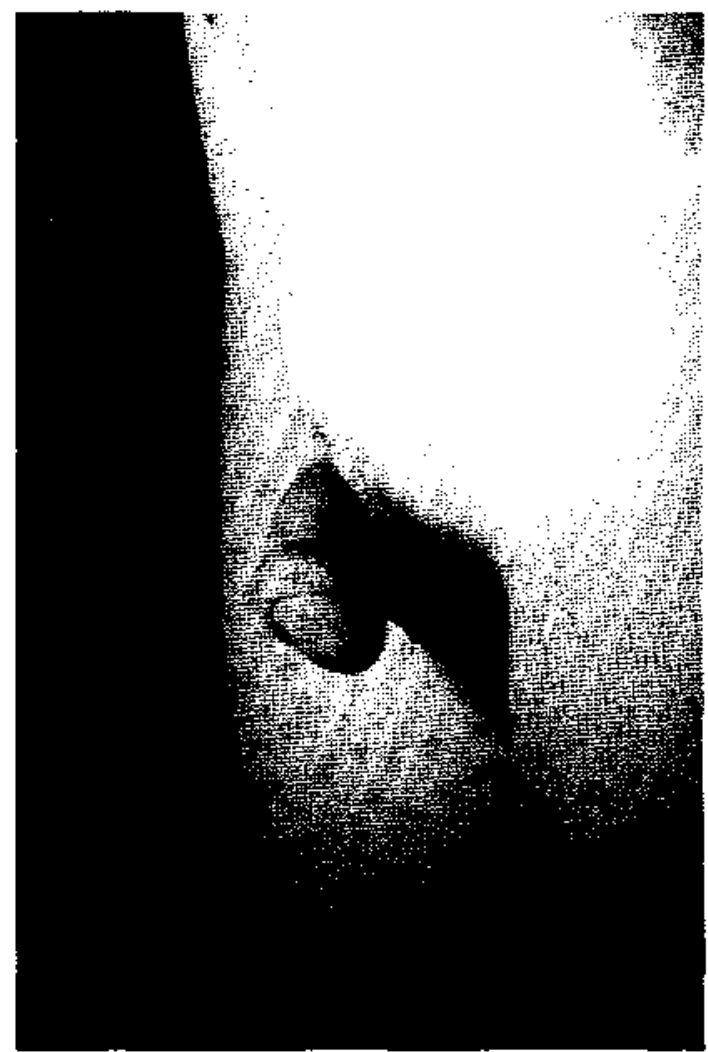

Figura 4: Virilización severa con pubertad precoz en una nin̄a de 5 años que se dejó como sexo masculìno.

doscopía y el genitograma permitieron clasificar el grado de virilización de las pacientes de acuerdo a los criterios de Prader ${ }^{1}$ en grados $2(n: 2), 3(n: 4), 4(n: 6), 5(n: 2)$ y $6(n: 1) . L a$ ecotomografía renal y la pielografía fueron normales en los casos en que se realizaron.

En 14 pacientes se hizo cirugía correctora para fenotipo femenino. Se practicaron cinco clitoridectcmías (antes de 1986), nueve plastías de clítoris entre las edades de un mes y 3,5 años; 14 plast ías vulvares junto con la cirugía clitoridiana y 14 plastías de introito vaginal entre un mes y los 19 años de edad. Se realizó un procedimiento de descenso vaginal en una niña de 9 años de edad por vía abdominal, interponiendo un trozo de ileon entre la vagina proximal y la vulva. En un caso que consultó a los 5 años y había sido criado como varón se acordó, previo estudio familiar y psiquiátrico, mantener ese sexo fenotípico, extirpándole ovarios, trompas, útero y vagina por vía abdominal (figura 4). 
Una paciente fue operada sin complicaciones al mes de edad, una vez estabilizado el trastorno hidroeléctrico. Se le realizó una plastia de clítoris, vulva e introjto vaginal. Evolucionó en perfectas condiciones pero falleció un mes más tarde por bronconeumonía aspirativa. Este es el único fallecimiento en la serie. Como complicaciones de la cirugía una paciente presentó infección urinaria que descompensó su equilibrio hidroeléctrico. Se trató con buenos resultados con antibióticos y aumento de la dosis de corticoides.

Los resultados de la cirugía fueron satisfactorios, con mejor estética en las pacientes en que se hizo plastía clitoridiana, vulvar y del introito vaginal en un tiempo operatorio.

El tiempo promedio de control postoperatorio para las niñas sometidas a clitoridectomía fue de 13 años y de 4 años en las que se hizo genitoplastia. Diez pacientes fueron controladas recientemente evaluando la estética vulvar, la amplitud y longitud vaginal, la sensibilidad cutánea del clítoris y la satisfacción personal o de la familia o ambas con el resultado quirúrgico. En todos los casos en que se corrigió la vagina de desembocadura baja en el seno urogenital se obtuvieron excelentes resultados, con introito adecuado independientemente de la edad de la operación. En las niñas mayores se midió el diámetro introital y vaginal con dilatadores de Heggar, encontrándose un diámetro suficiente para permitir relaciones sexuales. Una de ellas refirió haber tenido relaciones sexuales sin problemas. El resultado estético de la vulva y la sensibilidad táctil del clítoris parecieron adecuados. La uretra que inicialmente quedaba hipospádica demostró corregirse espontáneamente con el crecimiento, en las niñas mayores.

Tres pacientes requerian nueva cirugía: la niña en que se interpuso intestino (ileon) necesitará ampliación del introito vaginal en la etapa de actividad sexual; en otra la reducción del clítoris fue insuficiente y hay estenosis del introito vaginal, el que deberá ser ampliado quirúrgicamente. Finalmente el paciente al que se asignó sexo masculino necesitará prótesis testiculares con el fin de afirmar la identidad masculina y expandir el escroto que es muy plano. En la entrevista, la madre no se veía muy segura de la decisión de sexo, en la cual la familia fue taxativa. E1 niño conserva rasgos faciales y corporales de tipo feminoide, no así en su comportamiento.

\section{Comentario}

Históricamente, la clitoridectomia fue el método para tratar la hipertrofia del clítoris en njñas con HSVC. Los resultados tardíos tanto estéticos como funcionales no han sido satisfactorios $^{2-4}$. Una alternativa es la plastía clitoridiana ${ }^{3-8}$, la cual reduce el tamaño del clítoris, seccionando los cuerpos cavemosos sin extirpar el glande, al que se preserva su inervación e irrigación sanguínea. Para evitar este daño hemos disefiado una técnica que preserva la pared dorsal de los cuerpos cavernosos, sin necesidad de disecar individualmente el paquete vasculonervioso sobre él. Huffmann postula que el tamaño del clítoris no debe exceder de $4,3 \mathrm{~mm}$. Allen hace un estudio comparativo de tres técnicas quirürgicas (clitoridectomía, clitoroplastia y clitoroplastía reductiva), concluyendo que la clitoroplastía reductiva cumple con conservar la función psicosexual y da resultados cosméticos más satisfactorios ${ }^{5}$. En nuestros casos, aquellas pacientes intervenidas antes de $1986 \mathrm{se}$ les realizó una clitoridectomía y en los posteriores se hizo plastía clitoridiana.

Hay publicaciones recientes que coinciden en una reparación quinúrgica precoz, en las prime. ras semanas de vida ${ }^{5-7}, 9$. En un caso que operamos al mes de edad no tuvimos problemas técnicos en la intervención quiruirgica ni en el postoperatorio precoz. Desafortunadamente esta niña falleció por aspiración de leche un mes después de operada.

Con respecto a las intervenciones introitales hay diferencias de opinion en cuanto a la edad en que deben realizarse. Algunos autores ${ }^{5,8,9}$ encuentran deficientes los resultados cuando se han practicado en la edad preescolar, con estenosis de la vagina. Ellos sugieren que esta cirugía se haga después de la pubertad, ya que en esta etapa el seno urogenital y la vagina alta se hacen más prominentes y funcionales bajo la influencia de los estrógenos, lo que facilitaría la reparación. Sólo operarian precozmente si hay infecciones urinarias recurrentes por retención de orina en la vagina estenótica. Sin embargo, nos parece que estos trabajos se refieren a técnicas antiguas e insuficientes, ya que las solu. ciones quirúrgicas que proponen para las complicaciones son las mismas que ahora practicamos a una edad más temprana.

La mayoria actualmente plantea una reconstrucción genital completa y temprana. Donohue 
sugiere hacer la plastía clitoridiana, de labios y del introito vaginal en el período neonatal. Dos semanas después de la intervención se realizan dilataciones del nuevo introito vaginal, diariamente durante varias semanas, que luego se espacian hasta llegar a frecuencias de cada 6 meses. Ello con el fin de evitar la estenosis del introito que es la complicación más frecuente a largo plazo ${ }^{10}$.

Nos parece que la corrección precoz de la vulva y el clítoris evita problemas psicológicos y sociales a la familia y permite un desarrollo más armónico de la nifia en su sexo verdadero. La corrección vaginal puede hacerse precozmente, antes de los 6 meses, si la desembocadura de la vagina en el seno urogenital es baja. Idealmente hay que hacerla antes del alta de la hospitalización neonatal, si el enfermo está bien estabilizado. Esta cirugia podría necesitar retoques de introito en la época de actividad sexual. $\mathrm{Si}$ la desembocadura de la vagina es alta, la cirugia taginal se difiere hasta la edad escolar. Estos casos se abordan por vía abdominal, desinsertando la vagina de su comunicación con el seno urogenital, el que queda como uretra y se desciende la vagina al periné. Si ello no es posible por ser muy corta, se interpone un trozo de intestino que reemplaza la vagina distal faltante. Así se ha logrado una plastía que es funcional, permitiendo las relaciones sexuales y el embarazo de las pacientes.

Toda esta cirugia puede necesitar operaciones complementarias de ampliación del neointroito, que conviene debe diferir hasta que exista actividad sexual mantenida. La operación inicial cumple el objetivo de comunicar la vagina al exterior y permitir menstruaciones normales. Las dilata. ciones profilácticas del introito vaginal pueden ser muy traumáticas psicológicamente en la nifia prepúber a púber, por lo que disminuimos su uso a lo estrictamente necesario. Preferimos tratar un grado leve de estenosis introital, fácil de corregir, cuando comience la actividad sexual.

En la forma no perdedora de sal es indispensable el diagnóstico precoz para evitar la asignación de un sexo de crianza equivocado. Esto ocurrió en el paciente que consultó tardíamente por pubertad precoz y criptorquídea bilateral a los 5 años, el que debió quedar como sexo masculino. En el control tardio la madre no se vio muy segura de que la decisión tomada fue la adecuada, pero manifestó que el ambiente familiar y social fue el factor definitivo en de- cidir el sexo masculino de su hijo -ya que el paciente tenia 4 años y hubiera sido muy difícil en ese momento tomar una decisión distinta-, lo que parecía respaldado por el estudio psi. quiátrico y la experiencia en la literatura. Nos queda la duda si hubiera sido útil retrasar la extirpación de los genitales internos, con el fin de estar seguros de que la decisión tomada haya sido la adecuada. Para evitar errores en casos como el mencionado, debe hacerse una cromatina nuclear en todo recién nacido con criptorquídea, especialmente si es bilateral y si las gónadas no se palpan. Este procedimiento debiera estar disponible a lo largo de todo el pais. La reparación precoz de los genitales logra que los padres tengan bien definido el sexo de su hijo lo más tempranamente posible. Ello contribuye a crear un ambiente más adecuado para que el niño asuma el rol de su sexo y disminuye a ansiedad de los padres frente a sus hijos, familiares y amigos. Pensamos que también ello pueda contríbuir a un menor grado de abandono precoz del tratamiento supletorio hormonal por parte de los padres, situación que desgraciadamente aún existe en nuestro país, como ocurrió en una de las pacientes que controlamos recientemente. No sabemos cuánto de ello se deba al rechazo que puede originar una niña que no tiene aún sus genitales corregidos.

En las formas perdedoras de sal debe realizarse la cinugia una vez que el paciente se encuentre estabilizado desde el punto de vista metabólico y con una terapia hormonal suplementaria que haya demostrado ser adecuada para mantener al niño balanceado.

Debemos sentalar la importancia de la cistos. copía, previa a cualquier intervención quirúrgica. Con ella se ha logrado superar la calidad de la información que proporciona el genitograma, el cual involucra irradiación del enfermo y generalmente de las manos del operador. Sin embargo, hemos visto errores en el diagnóstico cistoscópico cuando la persona que lo realiza no está familiarizada con estas afecciones.

En el control tardio de 10 pacientes, encontramos buenos resultados estéticos. Nos llamó la atención el encontrar un diámetro introital suficiente, en la mayoría de los casos, y que la uretra tenía una desembocadura normal migrando, al parecer, desde su posición hipospádica a una posición anterior. Si bien el remanente clitoridiano era adecuado y con sensibilidad táctil en aquellos casos en que se prac- 
ticó una clitoroplastía, no sabemos si cumplirấ un papel sexual.

\section{Resumen}

Se presentan quince pacientes de hiperplasia suprarrenal virilizante controladas en el Hospital San Juan de Dios en los últimos 20 años. La 17 hidroxiprogesterona plasmática y pregnantriol urinario junto con la cromatina sexual positiva demostraron ser los exámenes de mejor sensibilidad para el diagnóstico. Los 17 cetoesteroides en orina no fueron diagnósticos en 2 casos. La evaluación preoperatoria para definir la anatomía mediante ecotomografía pelviana, genitograma y cistoscopia demostró ser útil en su conjunto, especialmente la cistoscopia si era realizada por manos expertas. La corrección quirúrgica precoz en la niñez demostró buenos resultados estéticos y funcionales en los enfermos que tenian más de dieciocho años en el control tardío. La cinugía vaginal con ampliación del introito demostró buen lumen en el control tardio, salvo un caso de interposición de intestino delgado, entre vagina alta y vulva, que requerirà más cirugía. En un caso en que se asignó el sexo masculino por diagnóstico tardío (a los 5 años), el niño ha tenido un buen desarrollo del sexo social pero conserva caracteres fenotipicos feminoides.

(Palabras claves: Hiperplasia suprarrenal congénita virilizante, genitoplastía.)

\section{Referencias}

1. Prader VA: Vollkommen Manliche aubere Genitalent wicklung und Salvetlust-syndrom bei Machden mit Koagenitalen adrenogenitalem Syndrome. Helv Paediat Acta 1958; $13: 5 \times 14$.

2. Mininberg DT: Phalloplasty in congenital adrenal hyperplasia. J Urol 1982; 128: 355-356.

3. Rajfer $J$, Ehrlich RM. Goodwin W'E: Reduction clitoroplasty via ventral approach. J Urol 1982; 128: 341-343.

4. Ansell $J S$, Rajfer $J$ : A new and simplified method for conceating the hypertrophied clitoris. J Pediatr Surg $1981 ; 16: 681-684$.

5. Allen LE, Hardy BE, Churchill BM: The surgical management of the enlatged clitoris. J Utrol 1982; 128: $351 \cdot 354$.

6. Sharp RJ, Holder TM. Howard PH et al.: Neonatal genital reconstruction. J Pediatr Surg 1987; 22: 168-171.

7. Oesserling $J E$, Gearhart $J P$, Jeffs $R D$ : A unified approach to early reconstructive surgery of the child with ambiguous genitalia. J Urol 1987; 138: 1079-1082.

8. Sotiropoulos A, Morishima A, Homsy $Y$ et al:; Long-term assessment of genital reconstruction in female pseudo hermaphrodites. J Urol 1976; 115: $599-601$.

9. Jones HW, Garcia SC, Klingensmith GI: Secondary surgical treatment of the masculinized external genitalia of patients with virilizing adrenal hyperplasia. Obstet Gynecol 1976; 48: 73-75.

10. Donahoe PK, Hendren WH: Perineal reconstruction in ambiguous genitalia infants raised as females. Ann Surg 1984; 198: 363-371. 